$M=$ man; MSA-C = Multiple System Atrophy - Cerebellar type; QUIP-RS Score = Questionnaire for ImpulsiveCompulsive Disorders in Parkinson's Disease-Rating Scale; SARA = Scale for the Assessment and Rating of Ataxia; Specific domain* ${ }^{*}$ Specific domain for impulsivity and compulsivity; SCA = Spinocerebellar Ataxia (genetically confirmed autosomal dominant ataxias); $\mathrm{W}=$ woman

\begin{tabular}{cccccc}
\hline Case & Diagnosis & $\begin{array}{c}\text { Duration of illness since } \\
\text { symptom onset (years) }\end{array}$ & CCAS* & $\begin{array}{c}\text { Beck depression } \\
\text { inventory II }\end{array}$ & $\begin{array}{c}\text { Barratt impulsiveness } \\
\text { scale }\end{array}$ \\
\hline Case 1 & SCA 2 & 22 years & $107 / 120 \& 0 / 10$ & $17 / 63$ & $4 / 120$ \\
Case 2 & SCA 3 & 6 years & $94 / 120 \& 0 / 10$ & $3 / 63$ & $72 / 120$ \\
Case 3 & SCA 1 & 11 years & $85 / 120 \& 2 / 10$ & $5 / 63$ & $71 / 120$ \\
Case 4 & SCA 2 & 4 years & $64 / 120 \& 6 / 10$ & $7 / 63$ & $47 / 120$ \\
Case 5 & MSA-C & 2 years & $74 / 120 \& 4 / 10$ & $20 / 63$ & $61 / 120$
\end{tabular}

${ }^{*}$ CCAS: cerebellar cognitive affective syndrome. The total score of the scale comprises two, one is the total raw score (total =120) and the other is the total pass score (pass of each domain. $=1$, failure of each domain $=0$, total $=10$ ); MSA-C = Multiple System Atrophy - Cerebellar type; SCA = Spinocerebellar Ataxia (genetically confirmed autosomal dominant ataxias)

\title{
Supplemental Table 1.
}

Cerebellar cognitive affective syndrome scale (CCAS-Scale), Beck depression index, and Barratt impulsiveness scale of the 5 individuals of the present case series. 